\title{
Diversidade de habitats físicos e sua relação com macroinvertebrados bentônicos em reservatórios urbanos em Minas Gerais
}

\author{
Joseline Molozzi ${ }^{1}$, Juliana S. França ${ }^{1}$, Thiago L. A. Araujo ${ }^{1}$, Tales H. Viana ${ }^{2}$, \\ Robert M. Hughes ${ }^{3} \&$ Marcos Callisto ${ }^{1}$
}

\footnotetext{
1. Laboratório de Ecologia de Bentos, Departamento de Biologia Geral, Instituto de Ciências Biológicas, Universidade Federal de Minas Gerais, Caixa Postal 486, Pampulha, Belo Horizonte, MG, Brasil, 30161-970. (http://www.biogeral.icb.ufmg.br/benthos/,jmolozzi@gmail.com)

2. COPASA - Companhia de Saneamento de Minas Gerais, Rua Mar de Espanha, 525, Santo Antônio, Belo Horizonte, MG, Brasil, 30330-900.

3. Department of Fisheries \& Wildlife, Oregon State University, Corvallis, Oregon, EUA.
}

\begin{abstract}
Diversity of physical habitat and its relationship with benthic macroinvertebrates in urban reservoirs in Minas Gerais. We assessed the effects of physical habitat structure on benthic macroinvertebrate communities in 3 reservoirs: Serra Azul (SA), Vargem das Flores (VF) and Ibirité (IB). We sampled physical and chemical habitat and benthic macroinvertebrates at 30 systematic sites in each reservoir. In SA, the dominant taxa out of 12 were Melanoides tuberculatus Müller, $1774(15.2 \%)$ and Chaoboridae (63.8\%). In VF, the dominant taxa, out of 11 were M. tuberculatus (34.2\%) and Oligochaeta (33.6\%). In IB, M. tuberculatus $(91.2 \%)$ and Chaoboridae (6.27\%) dominated and we collected only 7 taxa. In SA, benthos were significantly influenced by the deciduous shrub understory and bank angle. In IB and VF, the significant physical habitat variables were: land cover, human influence, and aquatic macrophytes. We conclude that human disturbances reduced terrestrial vegetation cover and riparian and littoral physical habitat structural complexity, leading to worse water quality. Those changes, in turn, reduced the taxonomic richness of the benthic macroinvertebrate communities.
\end{abstract}

KEYWORDS. Benthic fauna, habitat integrity, semi-lentic ecosystems, benthic bioindicators

RESUMO. Foram avaliados os efeitos da estrutura física de habitats em comunidades de macroinvertebrados bentônicos em três reservatórios: Serra Azul (SA), Vargem das Flores (FV) e Ibirité (IB). Mensurações de variáveis físicas e químicas e comunidade bentônica foram realizadas em 30 estações de amostragem em cada reservatório. Em SA, foram coletados 12 táxons com predomínio de Melanoides tuberculatus Müller, 1774 $(15,2 \%)$ e Chaoboridae (63,8\%). Em VF foram coletados 11 táxons sendo M. tuberculatus $(34,2 \%)$ e Oligochaeta (33,6\%) os dominantes. Em IB foram coletados 7 táxons com domínio de M. tuberculatus $(91,2 \%)$ e Chaoboridae $(6,27 \%)$. Em Serra Azul foi observada influência significativa do sub-bosque arbustivo e do ângulo de inclinação do barranco. Nos reservatórios de Ibirité e Vargem das Flores as variáveis significativas foram cobertura do solo, influência humana e macrófitas aquáticas. Concluímos que as ações humanas reduzem a cobertura da vegetação terrestre e a complexidade de habitats físicos na região litorâneos dos reservatórios, levando a uma redução na qualidade de água. Essas mudanças, por sua vez, reduzem a riqueza taxonômica dos macroinvertebrados bentônicos.

PALAVRAS-CHAVE. Fauna bentônica, integridade de habitats, ecossistemas semi-lênticos, bioindicadores bentônicos.

A ocupação desordenada de bacias hidrográficas, embora varie com a organização social em uma dada região, gera impactos e deteriora a qualidade das águas, alterando sua disponibilidade (CALlisto et al., 2005). Atividades antrópicas nas áreas de entorno contribuem para assoreamento de reservatórios urbanos, reduzindo a heterogeneidade de substratos, diminuindo a disponibilidade de habitats para a biota, com consequente perda de biodiversidade (Allan, 2004). As propriedades físicas e químicas da água, juntamente com as características no entorno dos reservatórios, influenciam a distribuição dos organismos aquáticos. Assim, a redução da diversidade de habitats físicos pode levar a uma simplificação das comunidades de organismos aquáticos (BUSCH \& LARY, 1996).

Alterações na composição e abundância de organismos aquáticos devem ser analisadas de acordo com os seus desvios para as comunidades esperadas em áreas com condições naturais de áreas de referência, ou com um "ambiente com um bom potencial ecológico" no caso de reservatórios (REYNOLDSON et al., 1997; REYNOLDSON \& WRight, 2000; Directive 2000/60/EC; STODDARD et al., 2006). Nos EUA, a United States Environmental Protection Agency (USEPA) elaborou um sistema de monitoramento utilizando um protocolo de avaliação de habitats físicos para lagos e rios (KAUFMANN \& WhitTier, 1997; USEPA, 2007).O protocolo baseia-se em três elementos-chave para avaliação em ecossistemas lênticos: (i) avaliação das variáveis físicas e químicas da água; (ii) avaliação da região litorânea, bosque e sub-bosque ribeirinho em um transecto de $25 \mathrm{~m}$ de observação; (iii) descrição das características ripárias e do litoral em todas as estações de amostragem (USEPA, 2007). Levantamentos de dados incluindo níveis de perturbações antrópicas, uso e ocupação do solo, aporte de nutrientes, tipo de substrato, entre outros, podem fornecer estimativas que, quando classificadas, ajudam a detectar estressores e a orientar programas de restauração de características físicas e químicas em reservatórios urbanos (KAUFMAnN \& WhitTIER, 1997). Quantificar os descritores estruturais é uma maneira de garantir maior confiabilidade às análises (KAUfMANN et al., 2008). Assim, modificações e adaptações do protocolo de diversidade de habitats desenvolvido para ecossistemas da América do Norte poderão contribuir como uma ferramenta integradora em programas de gestão de bacias hidrográficas brasileiras. O objetivo deste estudo foi avaliar a influência da complexidade estrutural dos habitats sobre as comunidades de macroinvertebrados bentônicos em reservatórios urbanos. 


\section{MATERIAL E MÉTODOS}

Foram amostrados três reservatórios urbanos na bacia hidrográfica do rio Paraopeba, afluente do rio São Francisco, Minas Gerais (Fig. 1).

O reservatório de Ibirité (2001'13.39"S; $44^{\circ} 06^{\prime} 44.88^{\prime \prime} \mathrm{W}$ ) está localizado a uma altitude de $773 \mathrm{~m}$. Apresenta acelerado processo de eutrofização artificial, o que se caracteriza por acentuada produção primária, com blooms de algas Microcystis sp. e de macrófitas aquáticas Eichhornia crassipes (CAllisto et al., 2005; MoReno \& Callisto, 2006). O reservatório de Vargem das Flores $\left(19^{\circ} 54^{\prime} 25.06^{\prime \prime} \mathrm{S} ; 44^{\circ} 09^{\prime} 17.78^{\prime \prime} \mathrm{W}\right)$, localiza-se a 838,64 $\mathrm{m}$ de altitude e apresenta ocupação urbana em suas margens. O reservatório de Serra Azul (1959'24.92”'S; $\left.44^{\circ} 20^{\prime} 46.74^{\prime \prime} \mathrm{W}\right)$ localiza-se a uma altitude de $760 \mathrm{~m}$ e juntamente com o reservatório de Vargem das Flores é considerado um importante manancial de abastecimento de água para a região metropolitana de Belo Horizonte (Morais et al., 2010).

Caracterização de habitats físicos. Foram realizadas avaliações semi-quantitativas nas zonas litorânea e ripária (bosque e sub-bosque) em 30 estações de amostragem numeradas de 1 a 30 em cada um dos três ecossistemas, totalizando 90 estações de amostragem. As coletas foram realizadas no período chuvoso de 2009 (dezembro), com nível de água mais

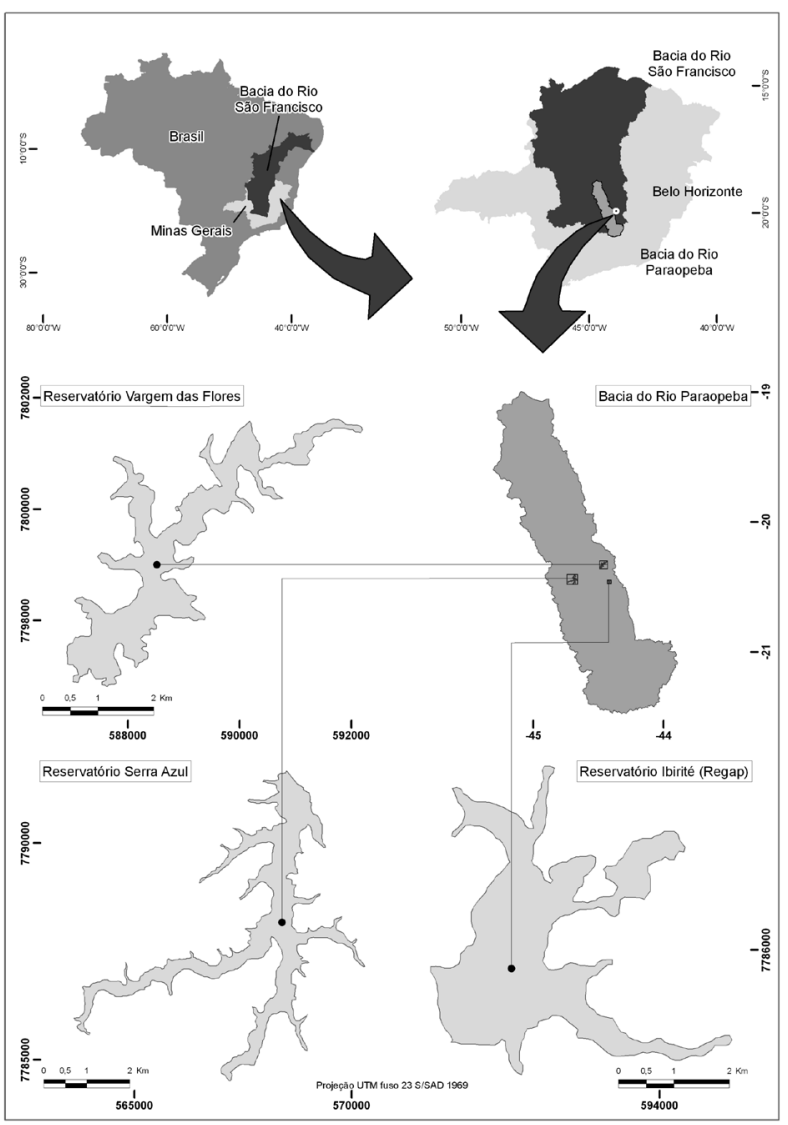

Fig. 1. Mapa com a localização dos reservatórios de Serra Azul, Vargem das Flores e Ibirité na bacia do Rio Paraopeba, Minas Gerais, Brasil. elevado e menor distância entre a lâmina d'água e a zona ripária. A posição da primeira estação amostral nos três reservatórios foi próxima ao vertedouro e as demais foram selecionadas com o intuito de abranger todo o reservatório, distribuídas regularmente ao longo da região litorânea. Em cada uma dessas estações foi determinada uma área com medidas pré-determinadas (10 $\mathrm{m}$ de comprimento - região litorânea e zona de transição, estendendo-se mais $15 \mathrm{~m}$ para a zona ripária - bosque e sub-bosque por $15 \mathrm{~m}$ de largura), conforme o US Environmental Protection Agency (BAKER et al., 1997; USEPA, 2007).

O protocolo de diversidade de habitats proposto por BAKER et al. (1997) e USEPA (2007) foi adaptado e modificado para utilização em reservatórios urbanos. Este protocolo é composto por variáveis categóricas (representadas por códigos/letras) ou classificadas em faixas percentuais $[0(>0-1 \%), 1(>1-10 \%), 2(>10$ $40 \%), 3(>40-75 \%)$ e $4(>75 \%)]$. O USEPA (2007) recomenda que complementar a aplicação do protocolo de diversidade de habitats seja mensurado variáveis físicas e químicas da água. A versão do protocolo traduzido e modificado para reservatórios urbanos encontra-se disponível no site do Laboratório de Ecologia de Bentos-UFMG (http://www.biogeral.icb. ufmg.br/benthos).

Composição granulométrica e teores de matéria orgânica nos sedimentos. A determinação da composição granulométrica dos sedimentos foi realizada segundo Suguio (1973), modificado por Callisto \& Esteves (1996) pela metodologia de peneiramento, onde uma alíquota de 100 gramas foi seca em estufa $\left(60^{\circ} \mathrm{C}\right.$ por 72 horas), e agitada em peneiras $(16,00 ; 4,00 ; 2,00 ; 1,00$; $0,50 ; 0,25 ; 0,125$ e $0,062 \mathrm{~mm})$. Os teores de matéria orgânica foram determinados pelo método de gravimetria onde alíquotas $(0,3 \pm 0,1 \mathrm{~g})$ foram calcinadas $\left(550^{\circ} \mathrm{C}\right.$ por $4 \mathrm{~h}$ ), pesadas e a diferença entre o peso inicial da amostra e o peso após a calcinação forneceu a porcentagem do teor orgânico das amostras de sedimento.

Variáveis físicas e químicas na coluna d'água. As coletas de água foram realizadas com garrafa do tipo Van D'orn na sub-superfície. As características físicas e químicas da água $[\mathrm{pH}$, condutividade elétrica $(\mu \mathrm{S}$ $\mathrm{cm}^{-1}$ ), turbidez (UNT), sólidos totais dissolvidos (mg $\left.\mathrm{L}^{-1}\right)$ ], temperatura da água $\left({ }^{\circ} \mathrm{C}\right)$ e oxigênio dissolvido ( $\mathrm{mg} \mathrm{L}^{-1}$ ) foram mensuradas in situ, utilizando-se um multianalisador modelo YSI. Leitura do disco de Secchi foi utilizada para avaliar a profundidade da zona eufótica (m). A profundidade da coluna d'água foi estimada com aparelho portátil do tipo Sonar (m).

Amostras de água foram transportadas para o laboratório de Ecologia de Bentos na Universidade Federal de Minas Gerais em frascos de polietileno e resfriadas para determinação dos teores de Nitrogênio total, Fósforo total e Ortofosfato, de acordo com "Standard methods for the examination of water and wastewater" (American Public Health Agency, 1992). 
Para a análise das concentrações de clorofila- $\alpha$ na coluna d'água, amostras de $500 \mathrm{~mL}^{-1}$ foram filtradas, com posterior maceração dos filtros (Millipore AP47) e extração em acetona fria $90 \%$ conforme Golterman et al. (1978).

Estrutura e composição de comunidades de macroinvertebrados bentônicos. Uma amostra de sedimento por estação de amostragem foi coletada com draga de Eckman-Birge $\left(0,0225 \mathrm{~m}^{2}\right)$ nos mesmos pontos de coleta onde foi utilizado o protocolo de diversidade de habitats. O material coletado foi fixado in situ com formol tamponado, acondicionado em sacos plásticos e transportado até o laboratório, onde as amostras foram lavadas em peneiras com abertura de malhas de 1,00 e 0,50 mm (LARSEN et al., 1991).

As larvas de Chironomidae foram clareadas com solução de lactofenol $10 \%$ e identificadas com o auxílio de um microscópio (aumento de 400 x).

Análises estatísticas. As diferenças nos valores dos parâmetros físicos e químicos entre os reservatórios foram testadas através de análise de variância simples (ANOVA one-way) (ZAR, 1999) com auxílio do programa STATISTICA 7.0. Para avaliar a estrutura das comunidades de macroinvertebrados bentônicos foram calculados o índice de diversidade de ShannonWiener, a densidade de organismos (indivíduos $\mathrm{m}^{-2}$ ) e a dominância de ocorrência $\left(\%\right.$ de indivíduos $\left.\mathrm{m}^{-2}\right)$.

Uma análise de agrupamento (Cluster) foi realizada para verificar a similaridade entre as estações de observações nos diferentes reservatórios. Esta foi realizada no software PAST versão 2.04 (HAMmER et al., 2001), utilizando o índice de distância de BrayCurtis e a UPGMA (Unweighted Pair Group Method with Arithmetic Mean) como método de amalgamação, sendo posteriormente avaliado o valor da correlação cofenética (HAMmer et al., 2001).

Análises de correspondência canônica (CCA) foram realizadas com os dados transformados para $\log (\mathrm{x}+1)$. Estas análises foram realizadas a fim de avaliar as principais tendências de variações dos dados entre a composição e estrutura da fauna bentônica, composição granulométrica dos sedimentos, variáveis físicas e químicas da água e as variáveis do protocolo de diversidade de habitats físicos para cada reservatório. Para a identificação da importância relativa das variáveis foi utilizado o modelo forward selection, que descreve as variáveis estatisticamente significativas, com nível de significância $\alpha$ de $95 \%(p<0,05)$. A significância das variáveis foi testada com 999 permutações. Das 68 variáveis mensuradas pelo protocolo de diversidade de habitats foram eliminadas as que não apresentaram variações significativas e as correlacionadas de forma redundante com outras variáveis no conjunto de dados, utilizando o programa CANOCO for Windows 4.5 (TER Braak \& Smilauer, 1998).

\section{RESULTADOS}

Caracterização da diversidade de habitats. A aplicação do protocolo no reservatório de Ibirité evidenciou alterações de origem antrópica em $46,6 \%$ das estações, variando de moderadas a severas. Nestes trechos a qualidade do ambiente está fortemente influenciada por atividades de pastagens, lixo, monocultura de Eucalyptus, residências, plantas industriais, lançamento de efluentes domésticos e industrial sem tratamento e controle de macrófitas aquáticas. Em Vargem das Flores somente $34,8 \%$ das estações de amostragem apresentaram distúrbios relacionados a atividades de camping, residências, pastagens, mineração, desmatamento e pesca (tanques rede). Em Serra Azul $10 \%$ das estações apresentaram alterações antrópicas em pequeno grau, sendo que o único distúrbio foi a presença de residências.

Em Serra Azul 100\% das estações apresentaram os maiores escores para as características "bem preservadas" e ambiente agradável. A zona ripária no entorno do reservatório é formado por árvores grandes, e o sub-bosque é constituído por arbustos. No reservatório de Ibirité $100 \%$ das estações apresentaram alto grau de distúrbio e ambiente desagradável. Além disso, observase intenso deflorestamento da zona ripária onde o subbosque é caracterizado por ervas altas e gramíneas. Em Vargem das Flores, 50\% das estações apresentaram os maiores escores para as características bem preservadas, as demais estações foram definidas como ambiente desagradável. Alguns trechos apresentam uma zona ripária com árvores grandes e um sub-bosque com arbustos, composto por plantas herbáceas e gramíneas.

Variáveis físicas e químicas na coluna d'água. As maiores variações na zona eufótica foram registradas nos reservatórios de Ibirité $(0,27 \pm 0,50 \mathrm{~m})$ e de Serra Azul $(2,5 \pm 0,63 \mathrm{~m})$ (Tab. I). Diferenças significativas foram encontradas entre os reservatórios de Serra Azul e Ibirité, para as variáveis abióticas; condutividade elétrica (ANOVA $\mathrm{F}_{2,58}=21,26 ; p=0,0001$ ), disco de Secchi (ANOVA $\mathrm{F}_{2,69}=14,80 ; \mathrm{p}=0,0001$ ), sólidos totais dissolvidos (ANOVA $\mathrm{F}_{2,58}=12,31 ; \mathrm{p}=0,005$ ), Nitrogênio total (ANOVA $F_{2,58}=44,42 ; p=0,0001$ ), Fósforo total (ANOVA $\left.\mathrm{F}_{2,58}=12,72 ; \mathrm{p}=0,0001\right) \mathrm{e}$ clorofila-a (ANOVA $\left.\mathrm{F}_{258}=12,04 ; \mathrm{p}=0,0002\right)$. As variáveis turbidez (ANOVA $\left.\mathrm{F}_{2,58}=21,39 ; \mathrm{p}=0,0001\right) \mathrm{e}$ Nitrogênio total (ANOVA $F_{2,69}=2,12 ; p=0,0001$ ) foram significativamente diferentes entre os reservatórios de Vargem das Flores e Ibirité. Não foram observadas diferenças significativas entre os reservatórios de Serra Azul e Vargem das Flores.

Composição granulométrica e teores de matéria orgânica nos sedimentos. No reservatório de Serra Azul houve predominância das frações areia média, areia fina e areia muito fina na maioria dos pontos amostrais (Tab. I). Em Vargem das Flores a fração cascalho foi observada em proporções inferiores a $1 \%$. No entanto, 
Tab I. Variáveis abióticas (média e desvio padrão), composição granulométrica (\%) e teores de matéria orgânica (\% P.S.) mensurados no mês de dezembro de 2009 nos reservatórios de Serra Azul, Vargem das Flores e Ibirité, bacia do Rio Paraopeba, Minas Gerais, Brasil.

\begin{tabular}{lccc}
\multicolumn{3}{c}{ Variáveis } & \multicolumn{3}{c}{ Reservatórios } \\
\cline { 2 - 4 } & Serra Azul & Vargem das Flores & Ibirité \\
\hline Pedras \% $(64-250 \mathrm{~mm})$ & $1,57 \pm 6,70$ & 0 & 0 \\
Cascalho \% $(2-64 \mathrm{~mm})$ & $1,36 \pm 5,17$ & $0,23 \pm 0,35$ & $0,25 \pm 0,47$ \\
Areia Grossa \% $(0,50-1,00 \mathrm{~mm})$ & $1,41 \pm 2,45$ & $14,30 \pm 8,72$ & $4,46 \pm 6,70$ \\
Areia Média \% $(0,250-0,50 \mathrm{~mm})$ & $15,68 \pm 8,25$ & $33,23 \pm 16,30$ & $22,15 \pm 11,51$ \\
Areia Fina \% $(0,125-0,250 \mathrm{~mm})$ & $19,09 \pm 10,03$ & $15,34 \pm 11,18$ & $25,26 \pm 9,38$ \\
Silte/argila \% $(<0,062 \mathrm{~mm})$ & $40,21 \pm 18,46$ & $17,98 \pm 11,62$ & $18,19 \pm 17,36$ \\
Matéria Orgânica $(\%$ P.S. $)$ & $12,24 \pm 3,19$ & $8,17 \pm 4,81$ & $8,10 \pm 5,03$ \\
Profundidade $(\mathrm{m})$ & $7,00 \pm 4,08$ & $3,89 \pm 1,56$ & $3,48 \pm 2,92$ \\
Discos de Secchi $(\mathrm{m})$ & $2,50 \pm 0,63$ & $1,05 \pm 0,28$ & $0,27 \pm 0,06$ \\
Temperatura do ar $\left({ }^{\circ} \mathrm{C}\right)$ & $32,00 \pm 0,45$ & $28,20 \pm 0,61$ & $28,67 \pm 1,53$ \\
Temperatura da água $\left({ }^{\circ} \mathrm{C}\right)$ & $30,20 \pm 0,44$ & $27,96 \pm 0,67$ & $27,96 \pm 0,67$ \\
pH & $8,14 \pm 0,44$ & $8,48 \pm 0,35$ & $9,01 \pm 0,50$ \\
Condutividade elétrica $\left.(\mu \mathrm{S} \mathrm{cm})^{-1}\right)$ & $26,13 \pm 4,07$ & $134,59 \pm 13,60$ & $285,81 \pm 38,03$ \\
Sólidos totais dissolvidos $\left(\mathrm{mg} \mathrm{L}^{-1}\right)$ & $16,77 \pm 4,49$ & $34,81 \pm 5,53$ & $177,35 \pm 22,03$ \\
Oxigênio Dissolvido $\left(\mathrm{mg} \mathrm{L}^{-1}\right)$ & $7,86 \pm 0,28$ & $7,56 \pm 0,63$ & $7,09 \pm 2,15$ \\
Turbidez $(\mathrm{UNT})$ & $1,43 \pm 0,53$ & $4,84 \pm 4,15$ & $23,70 \pm 4,22$ \\
Clorofila- $a\left(\mu \mathrm{g} \mathrm{L}{ }^{-1}\right)$ & $1,70 \pm 2,09$ & $3,00 \pm 2,98$ & $82,01 \pm 122,72$ \\
Nitrogênio total $\left(\mathrm{mg} \mathrm{L}^{-1}\right)$ & $0,06 \pm 0,01$ & $0,09 \pm 0,04$ & $0,26 \pm 0,06$ \\
Fósforo total $\left(\mu \mathrm{g} \mathrm{L}{ }^{-1}\right)$ & $58,96 \pm 25,53$ & $26,65 \pm 19,37$ & $114,20 \pm 50,94$ \\
Ortofosfato $\left(\mu \mathrm{g} \mathrm{L}^{-1}\right)$ & $9,09 \pm 8,84$ & $6,93 \pm 3,20$ & $12,22 \pm 21,58$ \\
\hline
\end{tabular}

silte e argila representaram mais que $30 \%$ da composição granulométrica dos sedimentos. Não foram observados blocos de pedras no reservatório de Ibirité e a fração cascalho foi registrada em proporções inferiores a 2,3\%. As frações de sedimento predominantes foram areia fina e areia muito fina, em $70 \%$ das estações. O sedimento é homogêneo em quase todas as estações e os teores de matéria orgânica não ultrapassaram $20 \%$ P.S., sendo que $55 \%$ das estações apresentaram valores inferiores a $10 \%$ P.S.

Estrutura e composição das comunidades de macroinvertebrados bentônicos. Foram coletados nos três reservatórios 831 organismos (Tab. II). A riqueza de táxons foi maior no reservatório de Serra Azul (12 táxons) seguida de Vargem das Flores (11) e Ibirité (7). A densidade total de indivíduos foi maior no reservatório de Ibirité $\left(84.400,0\right.$ ind $\left.\mathrm{m}^{-2}\right)$, seguida de Vargem das Flores (27.173,3 ind $\mathrm{m}^{-2}$ ) e Serra Azul (15.635,6 ind $\mathrm{m}^{-2}$ ). Dentre os grupos de macroinvertebrados, Melanoides tuberculatus Müller, 1774 representou 71,5\% dos organismos amostrados, seguido por Chironomidae $(19,1 \%)$.

No reservatório de Serra Azul foram coletados 116 indivíduos: 90,6\% larvas de Diptera, sendo que destas 63,8\% Chaoboridae. Dentre os Chironomidae, representantes de Chironominae (14,7\%) e Tanypodinae $(12,1 \%)$ foram encontradas nas amostras, com destaque para Coelotanypus (12,1\%), Procladius (5,2\%), Fissimentum (2,6\%), Aedokritus, Alotanypus e Harnischia (1,7\% cada).

Em Vargem das Flores foram coletados 205 indivíduos: Melanoides turberculatus (34,2\%), Oligochaeta (33,6\%), Corbicula fluminea Müller, 1774
$(5,9 \%)$ e Chironomidae $(21,4 \%)$, com destaque para Aedokritus (6,3\%) e Coelotanypus (2,4\%). No reservatório de Ibirité foram coletados 510 indivíduos, 91,2\% Melanoides turberculatus, 1,6\% Biomphalaria straminea Dunker, 1848 e 6,7\% Chironomidae, principalmente dos gêneros Coelotanypus e Chironomus, representando 0,2\% cada um (Tab. II).

A análise de agrupamento apresentou valor de correlação cofenética de $r=0,89$. Algumas estações apresentaram características de habitats semelhantes (estações 8, 11, 29, 9 e 28 em Serra Azul, e estações 29, 12 e 26 em Vargem das Flores) caracterizadas pela composição granulométrica dos sedimentos e por algumas características na região do entorno dos reservatórios. No entanto, não foi observado um padrão de diferenciação para os reservatórios na estrutura das comunidades, segundo a análise de Cluster (Fig. 2).

Os eixos 1 e 2 da CCA explicaram $68,7 \%$ da relação entre as principais variáveis do protocolo de caracterização da diversidade de habitats e as comunidades bentônicas no reservatório de Serra Azul. O eixo 1 explicou $39,4 \%$ da variação e as variáveis relacionadas negativamente foram clorofila- $a$, profundidade e sub-bosque, enquanto que as variáveis associadas positivamente foram a fração granulométrica areia fina e o ângulo de inclinação. A fração do sedimento areia fina explicou a relação entre o sedimento e a comunidade bentônica de forma significativa $(p=0,049)$. Das 13 variáveis abióticas utilizadas pelo protocolo de diversidade de habitats, clorofila- $a$ e profundidade foram significativas ( $p=0,015$ e $p=0,049$, respectivamente) (Fig. 3). As variáveis sub-bosque decidual herbácea e sub-bosque decidual arbusto, cobertura do solo, ervas 
Tab. II. Composição de macroinvertebrados bentônicos (média e desvio padrão) coletados no mês de dezembro de 2009 nos reservatórios de Serra Azul, Vargem das Flores e Ibirité, bacia do rio Paraopeba, Minas Gerais, Brasil.

\begin{tabular}{|c|c|c|c|}
\hline \multirow[t]{2}{*}{ Taxon } & \multicolumn{3}{|c|}{ Reservatórios } \\
\hline & Serra Azul & Vargem das Flores & Ibirité \\
\hline \multicolumn{4}{|l|}{ Mollusca } \\
\hline \multicolumn{4}{|l|}{ Gatropoda } \\
\hline \multicolumn{4}{|l|}{ Prosobranchia } \\
\hline \multicolumn{4}{|l|}{ Mesogastropoda } \\
\hline \multicolumn{4}{|l|}{ Thiaridae } \\
\hline Melanoides tuberculatus Müller, 1774 & $0,36 \pm 0,59$ & $2,50 \pm 3,90$ & $31,00 \pm 55,64$ \\
\hline \multicolumn{4}{|l|}{ Planorbiidae } \\
\hline Biomphalaria straminea Dunker, 1848 & & & $0,53 \pm 1,35$ \\
\hline \multicolumn{4}{|l|}{ Bivalvia } \\
\hline \multicolumn{4}{|l|}{ Corbiculidae } \\
\hline Corbicula fluminea Müller, 1774 & & $0,42 \pm 0,99$ & $0,06 \pm 0,25$ \\
\hline \multicolumn{4}{|l|}{ Annelida } \\
\hline Hirudinea & $0,05 \pm 0,22$ & $0,17 \pm 0,54$ & \\
\hline Oligochaeta & & $2,46 \pm 4,61$ & $0,13 \pm 5,79$ \\
\hline \multicolumn{4}{|l|}{ Insecta } \\
\hline \multicolumn{4}{|l|}{ Ephemeroptera } \\
\hline Polymirtacyidae & & $0,03 \pm 0,18$ & \\
\hline Baetidae & & $0,07 \pm 0,37$ & \\
\hline Leptoceridae & & $0,07 \pm 0,37$ & \\
\hline \multicolumn{4}{|l|}{ Odonata } \\
\hline \multicolumn{4}{|l|}{ Gomphidae } \\
\hline \multicolumn{4}{|l|}{ Diptera } \\
\hline \multicolumn{4}{|l|}{ Chaoboridae } \\
\hline Chaoborus Theobald, 1901 & $3,89 \pm 7,52$ & $0,89 \pm 2,88$ & $2,13 \pm 5,79$ \\
\hline Ceratopogonidae & $0,15 \pm 0,37$ & & \\
\hline \multicolumn{4}{|l|}{ Chironomidae } \\
\hline \multicolumn{4}{|l|}{ Tanypodinae } \\
\hline Coelotanypus Kieffer, 1913 & $0,73 \pm 1,24$ & $0,17 \pm 0,47$ & $0,06 \pm 0,25$ \\
\hline \multicolumn{4}{|l|}{ Chironominae } \\
\hline Aedokritus Roback, 1958 & $0,10 \pm 0,31$ & $0,46 \pm 1,75$ & \\
\hline Chironomus Meigen, 1803 & $0,05 \pm 0,22$ & & $0,06 \pm 0,25$ \\
\hline Fissimentum Cranston \& Nolte, 1996 & $0,10 \pm 0,30$ & & \\
\hline Tanytarsus van der Wulp, 1984 & & $0,03 \pm 0,18$ & \\
\hline Harnischia Kieffer, 1921 & $0,10 \pm 0,31$ & & \\
\hline Procladius Skuse, 1889 & $0,31 \pm 0,82$ & & \\
\hline Alotanypus Roback, 1971 & $0,10 \pm 0,31$ & & \\
\hline Parachironomus Lenz,1921 & $0,05 \pm 0,22$ & & \\
\hline
\end{tabular}

altas, gramíneas e ângulo de inclinação do barranco foram correlacionadas, sendo cobertura do solo a única variável significativa $(p=0,035)$.

No reservatório de Vargem das Flores, os eixos 1 e 2 explicaram $74,8 \%$ da relação entre as variáveis do protocolo e a comunidade bentônica. O eixo 1 explicou $41,6 \%$ da variabilidade a clorofila-a, abundância total de macrófitas aquáticas, e macrófitas aquáticas emergentes estiveram positivamente relacionadas. Ortofosfato e fração areia grossa foram relacionadas negativamente. Areia grossa explicou de forma significativa a relação entre as frações de sedimento e a fauna bentônica $(p=$ $0,049)$. Das variáveis abióticas, Ortofostato $(p=0,041)$ e clorofila- $\alpha(p=0,036)$ foram significativas. Tipo de cobertura $(p=0,023)$, tipo do ambiente ("near pristine") $(p=0,043)$, influência humana (comércio) $(p=0,022)$, influência humana (edifícios) $(p=0,013)$, zona de dossel (semi-decidual) $(p=0,015)$, abundância total de macrófitas aquáticas $(p=0,043)$, macrófitas aquáticas emergentes $(p=0,032)$, sub-bosque (com vegetação mista) $(p=0,049)$ e zona de transição (substrato composto por cascalho $)(p=0,043)$ foram significativas. No entanto, as únicas variáveis do protocolo que não apresentaram uma correlação redundante e por isso foram utilizadas na
CCA, foram abundância total de macrófitas aquáticas e macrófitas aquáticas emergentes (Fig. 4).

Os eixos 1 e 2 da CCA explicaram respectivamente $34,6 \%$ e $18,6 \%$ da variância dos dados, totalizando $53,2 \%$ da explicação compartilhada entre as variáveis do protocolo de diversidade de habitats e a fauna bentônica no reservatório de Ibirité. As variáveis clorofila- $a$ e Nitrogênio total relacionaram-se negativamente com o eixo 1, enquanto influência humana, tipo de cobertura e areia muito fina relacionaram-se positivamente. A areia muito fina explicou de forma significativa a relação entre a fauna bentônica e a composição granulométrica dos sedimentos. Ortofosfato $(p=0,019)$, clorofila- $a(p=$ $0,033)$, Nitrogênio total $(p=0,033)$ e oxigênio dissolvido $(p=0,012)$ foram significativas. As variáveis influência humana (pastagem) $(p=0,021)$, tipo de cobertura (vegetação) $(p=0,014)$, zona de dossel (ausência de vegetação) $(p=0,014)$, zona de transição (areia) $(p=$ $0,033)$, distúrbios humanos (moderados) $(p=0,049)$ e macrófitas aquáticas (flutuantes) $(p=0,049)$ foram significativas. As únicas variáveis que não apresentaram uma redundância na correlação e por este motivo foram utilizadas na CCA foram macrófitas aquáticas flutuantes e zona de dossel - ausência de vegetação (Fig. 5). 


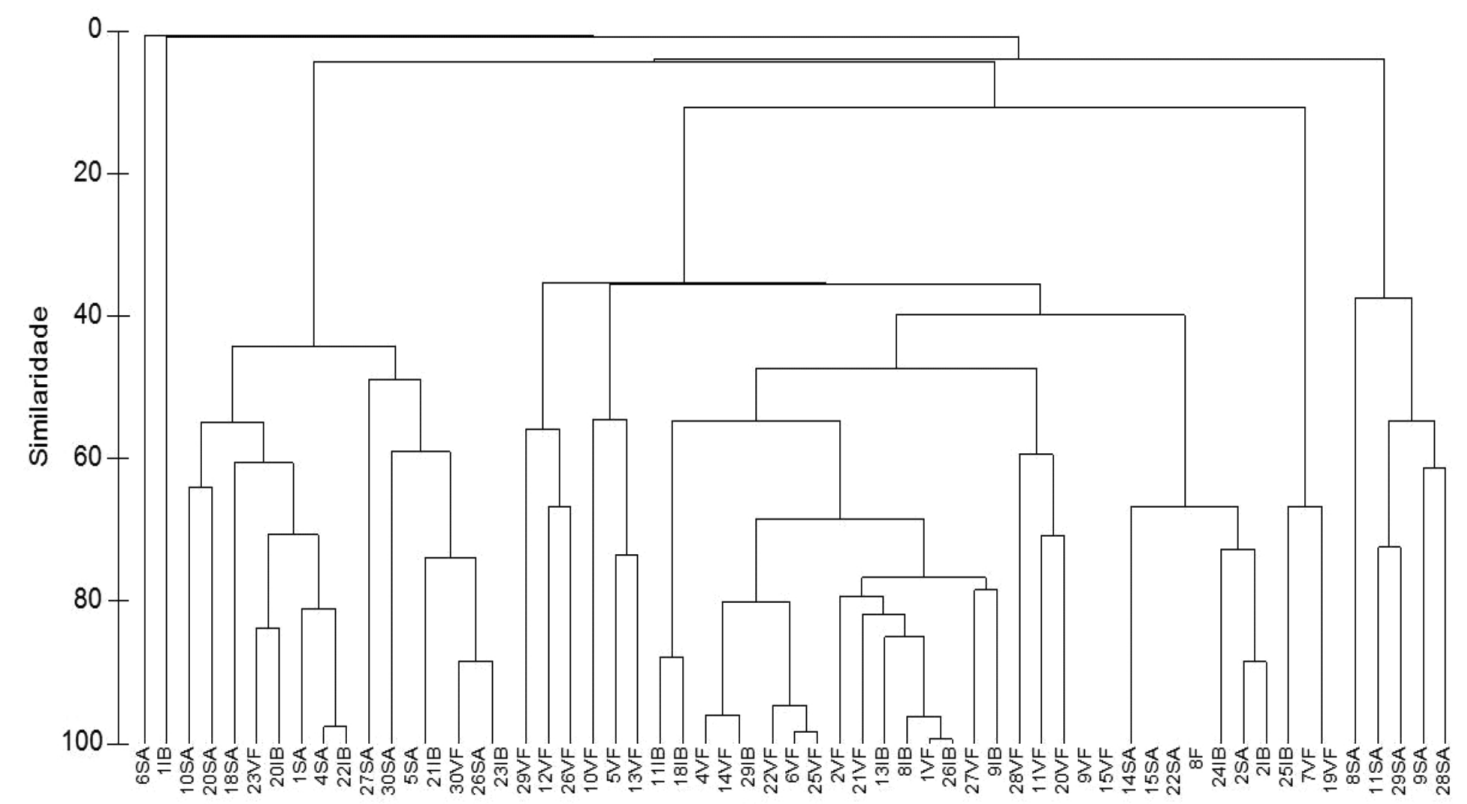

Fig. 2. Dendrograma de Similaridade da composição dos macroinvertebrados bentônicos nos reservatórios na bacia hidrográfica do rio Paraopeba, Minas Gerais, em dezembro de 2009. (SA, Serra Azul; VF, Vargem das Flores; IB, Ibirité).

\section{DISCUSSÃO}

No reservatório de Ibirité observa-se o processo de eutrofização artificial devido ao lançamento de esgotos domésticos sem tratamento (CALlisto et al., 2005), elevados teores de nutrientes na coluna d'água (Moreno \& Callisto, 2006), blooms de cianobactérias e macrófitas aquáticas flutuantes, baixos valores de visibilidade do disco de Secchi, provavelmente devido aos teores de matéria orgânica (RODGHER et al., 2005). O valor de fósforo total na água foi superior ao mensurado nos reservatórios de Serra Azul e Vargem das Flores. No entanto, em algumas estações de amostragem foram registrados valores acima do limite da Resolução CONAMA 357/2005, que estabelece que ambientes lênticos em condição de classe 2 apresentem concentrações de P-total na água inferiores a $30 \mu \mathrm{g} \mathrm{L}^{-1}$ (BRASIL, 2005).

Os elevados valores de condutividade elétrica registrados nos reservatórios de Vargem das Flores e Ibirité podem ser resultado do aporte de material carreado pelos rios à montante destes reservatórios. Lançamentos irregulares de efluentes industriais e/ou domésticos em bacias de drenagem refletem o nível de influência antrópica a que estes ecossistemas estão submetidos. Moreno \& CAllisto (2006), utilizando protocolo de caracterização ecológica rápida, verificaram avanço no processo de degradação na bacia hidrográfica do reservatório de Ibirité, com atividades agropastoris e lançamentos de esgoto não tratado e sedimentos diretamente para o reservatório, o que persiste até os dias atuais.

Em termos da composição granulométrica, a qualidade dos substratos em rios e lagos tem sido considerada como um dos fatores que mais influencia a distribuição de macroinvertebrados bentônicos. Esta abordagem foi desenvolvida com base em observações recentes de que alguns táxons de macroinvertebrados bentônicos são restritos a tipos de substratos; e que tipos diferentes de substratos são capazes de hospedar comunidades de invertebrados que diferem em biomassa, densidade total e riqueza (VITouseK, 1990). Um substrato mais diversificado oferece maior disponibilidade de habitats e microhabitats (em uma escala de indivíduos), alimentos (diretamente ou adsorvidos nas partículas do sedimento) e proteção (por exemplo, de correntes e predadores, como peixes bentônicos) (CARVALHO \& UIEDA, 2004). No presente estudo, a maior riqueza de organismos bentônicos foi verificada no reservatório de Serra Azul, onde foi registrada maior diversidade granulométrica nos sedimentos e o mínimo de perturbações na região litorânea.

$\mathrm{O}$ sedimento no reservatório de Ibirité é formado predominantemente por frações de areia fina e muito fina, caracterizando baixa heterogeneidade e disponibilidade de habitats físicos (Allan, 2004). Estudos em ecossistemas tropicais e temperados registraram altas densidades de oligoquetos e baixa diversidade de outros macroinvertebrados associada a substratos formados por siltes e areias (GALDEAn et al., 2000; Fenoglio \& Cucco, 2004). Assim, nossos resultados corroboram os estudos anteriores onde os oligoquetos foram encontrados associados a sedimentos formados por frações de areia e silte/argila ou areia fina, dominantes no reservatório de Vargem das Flores, onde predominaram sedimentos finos. A baixa riqueza de macroinvertebrados em sedimentos finos deve-se, provavelmente, a partículas muito próximas entre si e com menor conteúdo de água intersticial, reduzindo a captura de detritos de compostos orgânicos e a disponibilidade de oxigênio (Fenoglio \& Cucco, 2004). 


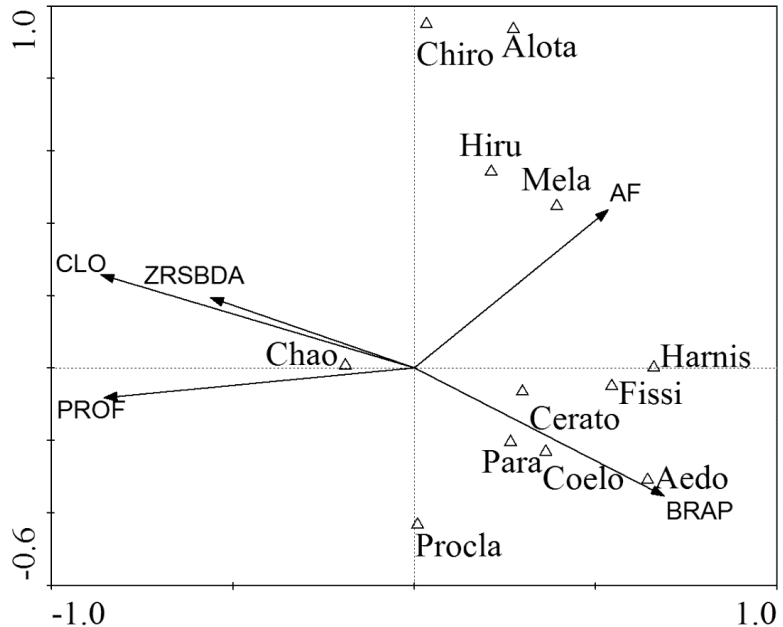

Fig. 3. Representação gráfica da Análise de Correspondência Canônica (CCA) entre composição do sedimento, variáveis físicas e químicas e variáveis de caracterização da diversidade de habitats relacionadas com os organismos bentônicos: Serra Azul; AF, areia fina (\%); BRAP, ângulo de inclinação do barranco (plano $<5 \%$ ); ZRSBDA, sub-bosque (decidual - arbustos); CLO, clorofila- $a\left(\mu \mathrm{g} \mathrm{L}^{-1}\right)$; PROF, profundidade (m); Aedo, Aedokritus; Alota, Alotanypus; Cerato, Ceratopogonidae; Coelo, Coelotanypus; Chiro, Chironomus; Fissi, Fissimentum; Harnis, Harnischia; Hiru, Hirudinea; Mela, Melanoides tuberculatus; Para, Parachironomus; Chao, Chaoboridae; Procla, Procladius.

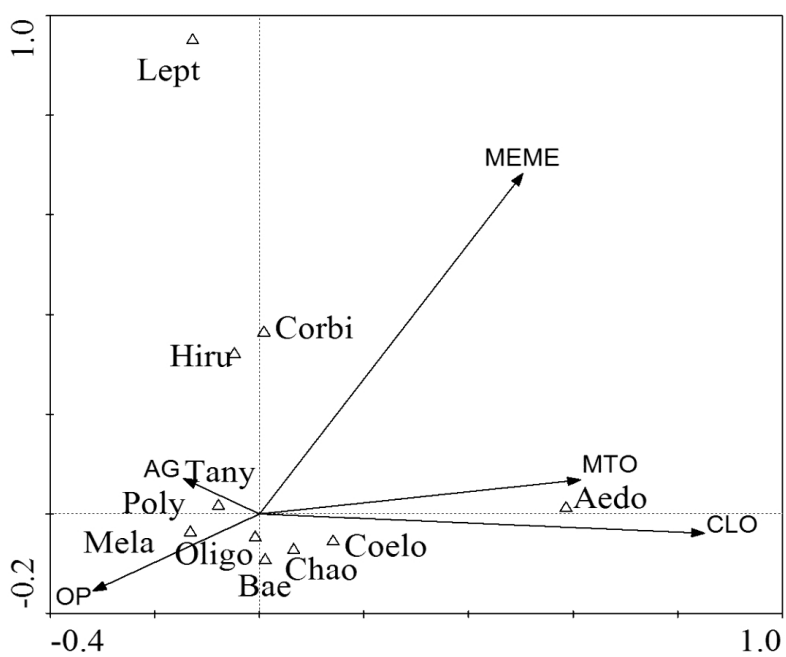

Fig. 4. Representação gráfica da Análise de Correspondência Canônica (CCA) entre composição do sedimento, variáveis físicas e químicas e variáveis de caracterização da diversidade de habitats relacionadas com os organismos bentônicos: Vargem das Flores; MEME, macrófitas aquáticas; MTO, abundância total de macrófitas aquáticas; AG, areia grossa (\%); OP, orto-fosfato $\left(\mu \mathrm{g} \mathrm{L}^{-1}\right)$; CLO, clorofila- $a\left(\mu \mathrm{g} \mathrm{L}^{-1}\right)$; Aedo, Aedokritus; Bae, Baetidae; Coelo, Coelotanypus; Corbi, Corbicula fluminea; Chao, Chaoboridae; Hiru, Hirudinea; Lept, Leptoceridae; Mela, Melanoides tuberculatus; Oligo, Oligochaeta; Poly, Polymitarcyidae; Tany, Tanytarsus.

Os oligoquetos de água doce vivem em todos os tipos de habitats, mas são mais abundantes em águas rasas, apesar de várias famílias terem representantes em lagos profundos (Alves et al., 2006).

Em Ibirité predominam Melanoides tuberculatus e Biomphalaria straminea. Estas são espécies exóticas e altamente competidoras que ameaçam a biodiversidade nestes ecossistemas (SouZA \& LimA, 1990). Indivíduos de $M$. tuberculatus são "r estrategistas" de reprodução partenogenética, com capacidade de manter altas densidades populacionais por longos períodos de tempo, além de possuírem grande capacidade migratória e fácil adaptação, estabelecendo-se em todos os tipos de substratos (FreITAS et al., 1987). Por estas razões são encontrados em diversos ecossistemas aquáticos, com diferentes graus de trofia e poluição, incluindo ambientes oligotróficos a hipereutróficos (Freitas et al., 1987; Rocha-Miranda \& Martins-Silva, 2006).

Os Chironomidae são os organismos mais representativos dos insetos aquáticos em decorrência da amplitude de ocupação de habitats; utilizam diversos recursos alimentares, oque confere estratégias adaptativas para colonizar diferentes tipos de micro-habitats por diferentes gêneros da família (Trivinho-Strixino \& SonADA, 2006). Essa ampla faixa de ocorrência em termos de substratos para os Chironomidae pode ser explicada por seus hábitos alimentares diversificados (ARmitage et al., 1994), podendo atuar como predadores, coletores de matéria orgânica particulada fina, e até eventualmente como fragmentadores (TuPINAMBÁs et al., 2007). De maneira geral, a família Chironomidae não apresenta exigências quanto ao substrato ideal para seu desenvolvimento (ENTREKIN et al., 2007).

Alguns gêneros registrados no reservatório de Vargem das Flores, como Tanytarsus, são habitantes de substratos arenosos sendo muito comuns em ambientes com baixo fluxo de água (RoQue et al., 2004), sendo considerados indicadores de locais com boa qualidade de água (PAnis et al., 1996). No reservatório de Serra Azul foram encontradas larvas de Fissimentum que, assim como Tanytarsus, são consideradas indicadoras de qualidade de água (LeAl et al., 2004; Morais et al., 2010). Por outro lado, em Ibirité foram registradas altas densidades e biomassa de Melanoides tuberculatus, que ocorre em número elevado em ecossistemas aquáticos impactados por atividades antrópicas (SILVA et al., 2010).

Quando avaliada a estrutura física dos habitats nos reservatórios, ficou evidente que algumas variáveis analisadas são extremamente importantes para a distribuição de comunidades bentônicas, tais como a influência humana, a presença de macrófitas aquáticas, o tipo do dossel e do subbosque da vegetação ripária. As alterações físicas decorrentes de atividades humanas (p. ex., deflorestamento da vegetação no entorno dos ecossistemas aquáticos provocado por atividades de agricultura, pecuária e urbanização) podem causar maior degradação aos ecossistemas aquáticos do que processos de eutrofização ou de acidificação para as comunidades aquáticas (BUSCH \& LARY, 1996).

As variáveis físicas mensuradas pelo protocolo evidenciam que algumas estações de amostragem podem apresentar potencial ecológico de referência nesta bacia hidrográfica (REYNOLDSON et al., 1997; StODDARD et al., 2006). As variáveis do protocolo de diversidade de habitats são medidas diretas ou indiretas do grau de conservação da diversidade de habitats que, por sua vez, podem favorecer 


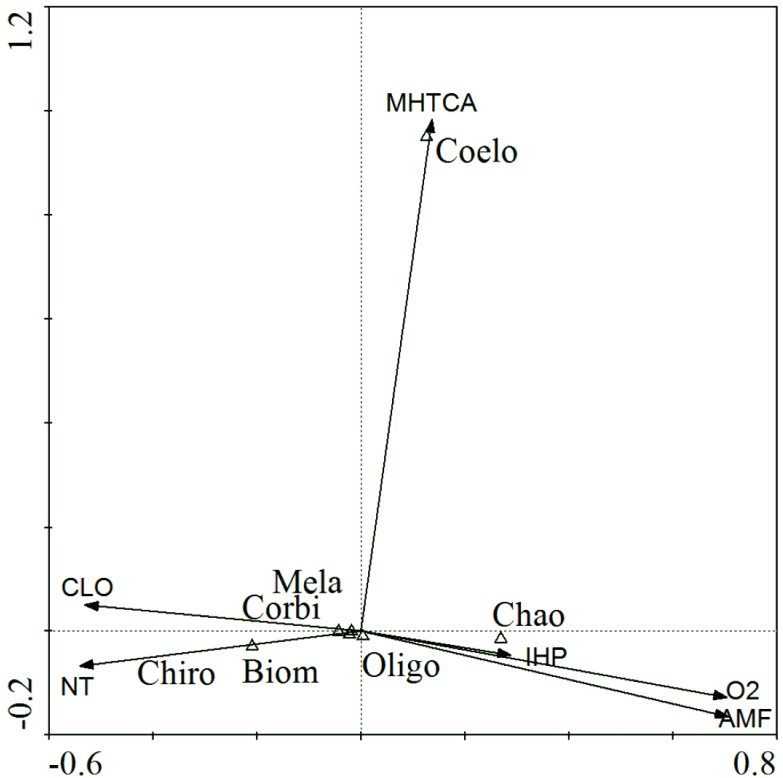

Fig. 5. Representação gráfica da Análise de Correspondência Canônica (CCA) entre composição do sedimento, variáveis físicas e químicas e variáveis de caracterização da diversidade de habitats relacionadas com os organismos bentônicos: Ibirité; MHTCA, tipo de cobertura (abundante); CLO, clorofila- $a$ ( $\left.\mu \mathrm{g} \mathrm{L}^{-1}\right)$; NT, nitrogênio total $\left(\mu \mathrm{g} \mathrm{L}^{-1}\right)$; $\mathrm{O}_{2}$, oxigênio dissolvido (mg L${ }^{-1}$ ); AMF, areia muito fina (\%); IHP, influência humana (pastagem); Biom, Biomphalaria straminea; Coelo, Coelotanypus; Corbicula fluminea; Chao, Chaoboridae; Chiro, Chironomus; Mela, Melanoides tuberculatus; Oligo, Oligochaeta.

uma elevada diversidade de macroinvertebrados (SuLLIVAN, 2006). No reservatório de Ibirité foi encontrada menor riqueza e elevada abundância de Melanoides tuberculatus, se comparado aos demais. Além disso, este reservatório caracterizou-se por apresentar substrato homogêneo, intensa ocupação humana e atividades no entorno que provocam distúrbios na estrutura física dos habitats aquáticos.

Em conclusão, o grau de conservação do entorno dos reservatórios é um fator de estrema importância para a distribuição da comunidade bentônica. Áreas com maior diversidade de habitats físicos oferecem abrigo e melhores condições para o estabelecimento de comunidades bentônicas. Nossos resultados evidenciam também a importância da utilização de um protocolo de avaliação da diversidade de habitats para relacionar com as comunidades demacroinvertebrados bentônicosemreservatóriosurbanos. Assim, acreditamos que as informações obtidas com a utilização do protocolo de diversidade de habitats poderá ser uma ferramenta complementar no monitoramento de ecossistemas semi-lênticos, auxiliando os órgãos gestores na detecção de estressores e assim ajudando a orientar programas de restauração das características físicas e químicas em reservatórios urbanos no Brasil.

Agradecimentos. Os autores agradecem à FAPEMIG e à CAPES pelas bolsas de doutorado e doutorado-sanduíche concedidas à JM; ao apoio financeiro e logístico oferecido em projetos e parcerias com FAPEMIG, CNPq, CAPES, PETROBRAS, COPASA, Projeto Manuelzão/UFMG, U. S. Fish and Wildlife Service e Fundação Fulbright. Aos colegas do Laboratório de Ecologia de Bentos, pelo apoio nas coletas de campo. Este manuscrito foi redigido enquanto MC estava em licença sabática (bolsa CAPES No. 4959/09-4) no IMAR, Universidade de Coimbra, Portugal.

\section{REFERÊNCIAS BIBLIOGRÁFICAS}

AlLan, J. D. 2004. Landscapes and riverscapes: the influence of land use on stream ecosystems. Annual Review of Ecology Evolution and Systematics 35:257-84.

Alves, R. G.; Marchese, M. R. \& Escarpinati, S. C. 2006. Oligochaeta (Annelida, Clitellata) in lotic environments in the state of São Paulo, Brazil. Iheringia, Série Zoologia 96(4):431-435.

Armitage, P.; Cranston, P. S. \& Pinder, L. C. V. eds. 1994. Chironomidae: Biology and Ecology of Non-biting Midges. London, Glasgow, New York, Tokyo, Melbourne, Madras. Chapman and Hall. 572p.

American Public Health Agency. 1992. Standard Methods for the Examination of Water and Wastewater. 18ed. Washington D.C., American Public Health Association.

Baker, J. R.; Peck, D. V. \& Sutton, D. W. eds. 1997. Environmental Monitoring and Assessment Program Surface Waters: Field Operations Manual for Lakes. EPA/620/R-97/001.U.S. Environmental Protection Agency. Corvallis, Oregon. 37p.

Brasil. 2005. Resolução n 357 de 17 de março de 2005 do Conselho Nacional do Meio Ambiente. Diário Oficial da União. Brasília, Seção 1, nº 53, 18 de março de 2005. p 58-63.

Busch, W. D. N. \& LARY, S. J. 1996. Assessment of habitat impairmentes impacting the aquatic of Lake Ontario. Canadian Journal of Fisheries and Aquatic Sciences 53(1):113-120.

Callisto, M. \& Esteves, F. 1996. Composição granulométrica do sedimento de um lago amazônico impactado por rejeito de bauxita e um lago natural. Acta Limnologica Brasiliensia 8:115-126.

Callisto, M.; Goulart, M.; Barbosa, F. A. R. \& Rocha, O. 2005. Biodiversity assessment of benthic macroinvertebrates along a reservoir cascade in the lower São Francisco river (Northeastern Brazil). Brazilian Journal of Biology 65(2):1-6.

Carvalho, E. M. \& Uieda, V. S. 2004. Colonização por macroinvertebrados bentônicos em substrato artificial e natural em um riacho da serra de Itatinga, São Paulo, Brasil. Revista Brasileira de Zoologia 21(2):287-293.

Directive 2000/60/EC. 2000. Water Framework Directive of the European Parliament and the Council, of 23 October 2000, establishing a framework for Community action in the field of water policy. Journal of the European Communities L327:172.

Entrekin, S. A.; Wallace, J. B. \& Eggert, S. L. 2007. The response of Chironomidae (Diptera) to a long-term exclusion of terrestrial organic matter. Hydrobiologia 575:401-413.

Fenoglio, S; Bo, T. \& Cucco, M. 2004. Small-scale macroinvertebrate distribution in a riffle of a neotropical rainforest stream (Rio Bartola, Nicaragua). Caribbean Journal of Science 40(2):253257.

Freitas, J. R.; Bedê, L. C.; Marco Júnior, P.; Rocha, L. A. \& Santos, M. B. 1987. Population dynamics of aquatic snails in Pampulha Reservoir. Memórias do Instituto Oswaldo Cruz 82(4):299-305.

Galdean, N.; Callisto, M. \& Barbosa, F. 2000. Lotic ecosystems of Serra do Cipó, southeast Brazil: water quality and a tentative classification based on the benthic macroinvertebrate community. Aquatic Ecosystem Health and Management 3(4):545-552.

Golterman, H. L.; Clymo, R. S. \& Ohnstad, M. A. M. 1978. Methods for physical and chemical analisys of freshwaters. 2ed. Oxford, Blackwell Scientific Publications (IBP 8). 213p.

Hammer, O.; Harper, D. A. T. \& Ryan, P. D. 2001. PAST: Paleontological Statistics Software Package for Education and Data Analysis. Palaeontologia Electronica 4(1). Disponível em: <http://palaeo-electronica.org/2001_1/past/issue1_01.htm>. Acesso em/Assessment: 09.11.2010.

Kaufmann, P. R. \& Whittier, T. R. 1997. Habitat characterization. In: Baker, J. R.; Peck, D. V. \& Sutton, D. W. eds. Environmental Monitoring and Assessment Program Surface Water: Field Operations Manual for Lakes. EPA/620/r-97/001, US. Environmental Protection Agency: Corvallis, OR: 5(1):5-26.

Kaufmann, P. R.; Faustini, J. M.; Larsen, D. P. \& Shirazi, M. A. 2008. A roughness-corrected index of relative bed stability for regional stream surveys. Geomorphology 99:150-170.

Larsen, D. P.; Stevens, D. L.; Selle, A. R. \& Paulsen, S. G. 1991 Environmental Monitoring and Assessment Program: EMAPSurface Waters, A northeast lakes pilot. Lake and Reservoir Management 7:1-11. 
Leal, J. J. F.; Esteves, F. A. \& Callisto, M. 2004. Distribution of Chironomidae larvae in an Amazonian flood-plain lake impacted by bauxite tailings (Brazil). Amazoniana 18(1/2):109-123.

Maroneze, D. M.; Tupinambás, T. H.; Alves, C. B. M.,; Vieira, F.; Pompeu, P.S. \& Callisto, M. 2011. Fish as ecological tools to complement biodiversity inventories of benthic macroinvertebrates. Hydrobiologia. 673(1):29-40.

Morais, S.; Molozzi, J.; Lessa, A.; Viana, T. H. \& Callisto, M. 2010. Diversity of larvae of littoral Chironomidae (Diptera-Insecta) and their role as bioindicators in urban reservoirs of different trophic levels. Brazilian Journal of Biology 70(4):995-1004.

Moreno, P. \& CAllisto, M. 2006. Benthic macroinvertebrates in the watershed of an urban reservoir in southeastern Brazil. Hydrobiologia 560:311-321.

Panis, L. T.; Goddeeris, B. \& Verheyen, R. 1996. On the relationship between vertical microdistribuition and adaptations to oxygen stress in littoral Chironomidae (Diptera). Hydrobiologia 318:6167.

Reynoldson, T. B.; Norris, R. H.; Resh, V. H.; Day, R. H. \& RosenberG, D. M. 1997. The reference condition: a comparison of multimetric and multivariate approaches to assess water-quality impairment using benthic macro invertebrates. Journal of the North American Benthological Society 16:833-852.

Reynoldson, T. B. \& Wright, J. F. 2000. The reference condition: problems and solutions. In: Wright, J. F.; Sutcliffe, D. W. \& Furse, M. T. eds. Assessing the biological quality of fresh waters: RIVPACS and others techniques. Freshwater Ambleside, Cumbia, Biological Association. p.293-303.

Rocha-Miranda, F. \& Martins-Silva, M. J. 2006. First record of the invasive snail Melanoides tuberculatus (Gastropoda: Prosobranchia: Thiaridae) in the Paranã river basin, GO, Brazil. Brazilian Journal of Biology 66(4):1109-1115.

Rodgher, S.; Espíndola, E. L. G.; Rocha, O.; Fracácio, R.; Pereira, R. H. G. \& Rodrigues, M. H. 2005. Limnological and ecotoxicological studies in the cascade of reservoirs in the Tietê river (São Paulo, Brazil). Brazilian Journal of Biology 65(4):697-710.
Roque, F. O.; Cororreia, L. C. S.; Trivinho-Strixino, S. \& Strixino, G. 2004. A review of Chironomidae studies in lentic system in the state of São Paulo, Brazil. Biota Neotropica 4:1-19.

Silva, E. C.; Molozzi, J. \& Callisto, M. 2010. Size-mass relationships of Melanoides tuberculatus (Thiaridae: Gastropoda) in a eutrophic reservoir. Zoologia 27(5):691-695.

Souza, C. P. \& Lima, L. C. 1990. Moluscos de Interesse Parasitológico do Brasil, Série Esquistossomose 1. Belo Horizonte, Centro de Pesquisas René Rachou, Fundação Oswaldo Cruz. 76p.

Stoddard, J. L.; Larse, D. P.; Hawkins, C. P.; Jonson, R. K. \& Norris, R. H. 2006. Setting expectations for the ecological conditions of streams: the concept of reference condition. Ecological Applications 16:1267-1276.

Suguio, K. 1973. Introdução à sedimentologia. São Paulo, Edgard Blücher, EDUSP. 317p.

Sullivan, C. A. 2006. Freshwater biodiversity: importance, status and conservation challenges. Biological Review 81:163-182.

Ter Braak, C. J. F. \& Smilauer, P. 1998. Canoco for Windows V. 4.5. Micro computer Power, Ithaca, New York.

Trivinho-Strixino, S. \& Sonada, K. C. 2006. A new Tanytarsus species (Insecta, Diptera, Chironomidae) from São Paulo State, Brazil. Biota Neotropica 6(2):1-9.

Tupinambás, T. H.; Callisto, M. \& Santos, G. B. 2007. Benthic Macroinvertebrate assemblage structure in two headwather streams, south eastern, Brazil. Revista Brasileira de Zoologia 24(4):887-897.

USEPA. 2007. Evaluation of the Nation's Lakes: Field Operations Manual. EPA 841-B-07-004. Washington D.C., E.S Environmental Protection Agency. 332p.

VitouseK, P. M. 1990. Biological invasions and ecosystem processes: towards integration of population biology and ecosystem studies. Oikos 57:7-13.

ZAR, J.H. 1999. Biostatistical analysis. $4^{\mathrm{a}}$ ed. New Jersey, PrenticeHall. 663p 